\title{
The evolution of close helium star to neutron star binaries
}

\author{
Jasinta D.M. Dewi \\ Sterrenkundig Insituut Anton Pannekoek, Universiteit van Amsterdam, \\ Kruislaan 403, NL-1098 SJ Amsterdam, Nederland, \\ and \\ Observatorium Bosscha and Jurusan Astronomi ITB, \\ Lembang 40391, Jawa-Barat, Indonesia \\ Onno R. Pols \\ Sterrekundig Instituut, Universiteit Utrecht, \\ Princetonplein 5, NL-3584 CC Utrecht, Nederland
}

\begin{abstract}
We present the evolution of helium stars in binary systems with a $1.4 \mathrm{M}_{\odot}$ neutron-star companion which will produce double neutron-star binaries, i.e., systems with helium stars more massive than $2.5 \mathrm{M}_{\odot}$. We found that mass transfer from helium star less massive than $\sim 3.3 \mathrm{M}_{\odot}$ will end up in a common-envelope phase. If the neutron star has enough time to complete the spiral-in before the core of the helium star collapses, the system will become a very tight double neutron-star binary $(P \approx 0 \mathrm{~d} 01)$. More massive helium stars do not go through a dynamically-unstable mass transfer. The outcome of binaries with helium star in this range of mass is double-neutron star systems with period of $0.1-1^{d}$, suggesting them to be the progenitor of the observed Galactic double neutron-star pulsars B $1913+16$ and B $1534+12$. Wide DNS pulsars like $\mathrm{J} 1518+4904$ are produced from helium star-neutron star binaries which avoid Roche-lobe overflow. We are also able to distinguish the progenitors of Type Ib supernovae (as the high-mass helium stars or systems in wide orbits) from those of Type Ic supernovae (as the lower-mass helium stars or systems in close orbits).
\end{abstract}

In this work we study the evolution of helium stars with masses of $2.8-6.7 \mathrm{M}_{\odot}$ with a $1.4 \mathrm{M}_{\odot}$ neutron-star companion. Such systems are assumed to be the remnants of $\mathrm{Be} / \mathrm{X}$-ray binaries with masses in the range of $10-20 \mathrm{M}_{\odot}$ which underwent mass transfer as case B (Roche lobe overflow is initiated during hydrogen shell burning) or case $\mathrm{C}$ (during helium shell burning), and survive a common-envelope and spiral-in phase. For a detailed explanation we refer to Dewi et al. (2002) and Dewi \& Pols (in preparation).

At the end of the evolution of lower mass helium stars $\left(M_{\mathrm{He}} \lesssim 3.3 \mathrm{M}_{\odot}\right)$, a helium convective envelope develops and mass-transfer rate increases rapidly; i.e., mass transfer becomes dynamically unstable leading to a common-envelope phase. If there is enough time for the neutron star to complete the spiral-in process before the core of helium star collapses, the system will produce very tight double neutron stars with $P=0$ d.01, with a merging timescale of $\sim 10^{5} \mathrm{yr}$. Otherwise, the core collapses while the neutron star is still in the envelope of helium star. 

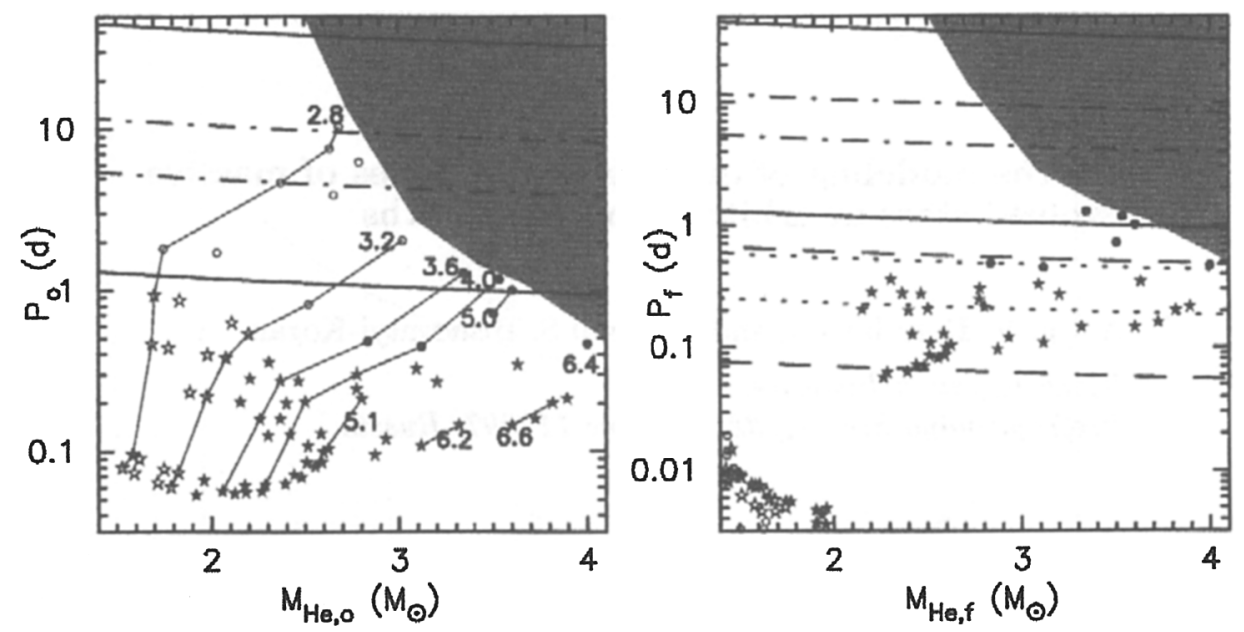

Figure 1. The range of period of the pre-SN progenitor of PSRs B 1913+16 (dashed-), B 1534+12 (dotted-), J 1518+4904 (dash-dotted-), and J 1811-1736 (solid-line). The shaded area marks the region where a helium star evolves without a period of RLOF. The stars and circles represent the remnants of case $\mathrm{BB}$ and case $\mathrm{BC}$ evolution, respectively. The solid symbols represent the remnants of $M_{\mathrm{He}}>3.3 \mathrm{M}_{\odot}$ stars, and the open ones indicate the remnants of lower-mass helium stars. Thin lines connect the remnants of helium stars with the same initial mass $M_{\mathrm{i}}$. Left: the mass and period before the spiral-in phase (after RLOF). Right: the mass and period after the spiral-in phase (prior to SN explosion).

The development of helium convective envelope is not found in the more massive helium stars, and therefore we suggest that they do not undergo a common-envelope phase. Systems with more massive helium stars produce double neutron stars with the orbit of $0 d^{d} 1-1^{d}$, suggesting them to be the possible progenitors of B $1913+16$ and B $1534+12$. The wide orbit J 1518+4904-like systems are produced from helium stars which avoid mass-transfer phase altogether.

The final amount of helium left in the envelope probably determines whether the explosion will be observed as a Type Ib or a Type Ic SN. The main observational criterion to distinguish between these types is the presence of helium in TypeIb and its absence in TypeIc SN. After the phase of mass transfer, the amount of helium in the envelope before the supernova explosion is smaller in systems with lower mass and shorter period. Therefore, we conclude that systems with higher-mass helium star or in wide orbits are the progenitors of Type Ib supernovae, and those with lower-mass helium star or in close orbits are the progenitors of Type Ic.

Acknowledgments. This work was sponsored by NWO Spinoza Grant 080 to E.P.J. van den Heuvel. JDMD thanks the Leids Kerkhoven-Bosscha Fonds for a travel grant.

\section{References}

Dewi, J.D.M., Pols, O.R., Savonije, G.J., van den Heuvel, E.P.J. 2002, MNRAS 331, 1027 